\title{
TOTALLY DISCRETE EXPLICIT AND SEMI-IMPLICIT EULER METHODS FOR A BLOW-UP PROBLEM IN SEVERAL SPACE DIMENSIONS.
}

\author{
PABLO GROISMAN
}

\begin{abstract}
The equation $u_{t}=\Delta u+u^{p}$ with homegeneous Dirichlet boundary conditions has solutions with blow-up if $p>1$. An adaptive time-step procedure is given to reproduce the asymptotic behvior of the solutions in the numerical approximations. We prove that the numerical method reproduces the blow-up cases, the blow-up rate and the blow-up time. We also localize the numerical blow-up set.
\end{abstract}

\section{INTRODUCTION.}

We study the behavior of an adaptive time step procedure for the following parabolic problem

$$
\left\{\begin{array}{lr}
u_{t}=\Delta u+u^{p} & \text { in } \Omega \times[0, T), \\
u(x, t)=0 & \text { on } \partial \Omega \times[0, T), \\
u(x, 0)=u_{0}(x)>0 & \text { on } \Omega .
\end{array}\right.
$$

Here $p$ is superlinear $(p>1)$ in order to have solutions with blow-up. We assume $u_{0}$ is regular and $\Omega \subset \mathbb{R}^{d}$ is a bounded smooth domain in order to guarantee that $u \in C^{2,1}$. A remarkable fact in this problem is that the solution may develop singularities in finite time, no matter how smooth $u_{0}$ is. For many differential equations or systems the solutions can become unbounded in finite time (a phenomena that is known as blow-up). Typical examples where this happens are problems involving reaction terms in the equation like (1.1) where a reaction term of power type is present and so the blow-up phenomenum occurs in the sense that there exists a finite time $T$ such that $\lim _{t \rightarrow T}\|u(\cdot, t)\|_{\infty}=+\infty$ if the initial data is large enough (see $[24,25]$ and references therein). The blow-up set, which is defined as the set composed of points $x \in \Omega$ such that $u(x, t) \rightarrow+\infty$ as $t \rightarrow T$, is localized in thin regions of $\Omega$, in [28] is proved that the $(d-1)$ dimensional Hausdorff measure of the blow-up set is finite. The blow-up rate at these points is given by $u(x, t) \sim(T-t)^{-\frac{1}{p-1}}$, moreover

$$
\lim _{t \rightarrow T}(T-t)^{\frac{1}{p-1}}\|u(\cdot, t)\|_{L^{\infty}(\Omega)}=C_{p}, \quad C_{p}=\left(\frac{1}{p-1}\right)^{\frac{1}{p-1}}
$$

Key words and phrases. Blow-up, adaptive numerical scheme, asymptotic behavior. 2000 Mathematics Subject Classification. 65M60, 65M20, 35K60, 35B40.

Partially supported by Universidad de Buenos Aires under grant X066, by ANPCyT PICT No. 03-00137 and Fundación Antorchas. 
(see $[16,17])$.

Hereafter we use the notation $f(t) \sim g(t)$ to mean that there exist constants $c, C>0$ independent of $t$ such that

$$
c g(t) \leq f(t) \leq C g(t)
$$

We remark that these results hold if $p$ is subcritical $\left(p<\frac{d+2}{d-2}\right.$ if $\left.d \geq 3\right)$. For supercritical $p$ the solutions may present different behaviors. For that reason we assume $1<p<\frac{d+2}{d-2}$ along the rest of the paper. However the asymptotic properties of the numerical schemes described above hold for every $p>1$, this is a difference between the continuous solutions and their approximations.

Since the solution $u$ develops a singularity in finite time, it is relevant to study the asymptotic behavior (close to the blow-up time) of numerical approximations for this kind of problems.

The first works that address this topic are [22, 23], where the authors analyze finite differences schemes for problem (1.1) with $\Omega=(0,1)$ and $p=2$. They study totally discrete schemes with a uniform spatial mesh and they adapt the time step with an explicit Euler method. They prove that in case that both the numerical approximations and the continuous solution blow up, the numerical blow-up times converge to the continuous one as the parameter of the method goes to zero.

A similar result is proved in [11] for the more general case $p>1$ and also a propagation result is shown: if the initial datum is symmetric and increasing in $[0,1 / 2]$ then $x=1 / 2$ is the only blow-up point. It is proved that if $x=1 / 2$ is a point of the mesh then this is the only numerical blow-up point if $p>2$, but if $p=2$ the blow-up propagates to the adjacent nodes (the adjacent nodes also blow up).

An adaptive in space algorithm is developed in [7]. This method refines the mesh as time goes forward using the scale invariance of solutions to this equation. The authors use this scheme to conjecture a second term in the asymptotic expansion of the solution.

In $[8,9,10,20]$ the so called moving mesh methods are developed. They also make use of the scaling invariance. They use a spatial mesh that is modified as time goes forward. The nodes are moved according to a moving mesh partial differential equation in such a way that the mass is uniformly distributed at any time. Although these methods are frequently used, they just work in the one dimensional case.

Semidiscrete schemes are considered in $[2,3]$ for $\Omega=(0,1)$. The spatial variable is discretized while time remains continuous. The authors prove convergence of the method in regions where the solution is regular as well as conditions that ensure the presence of blow-up in the numerical scheme $(p>1$ and some hypotheses on the initial data). They also prove convergence of the numerical blow-up times in some situations.

The same scheme is considered in [18], where the authors find the blow-up rate and the blow-up set of the numerical solutions and prove that they reproduce the 
theoretical ones. They also prove that the numerical solutions blow up if the theoretical one does and if the parameter of the method is small enough. Convergence of the blow-up times is also proved without any further assumptions.

In $[4,14]$ the authors consider the heat equation in an interval with a nonlinear Neumann condition at the boundary. They find conditions that guarantee the presence of blow-up in the numerical approximations (that differs with the ones for the continuous problem) and convergence of the method and the numerical blow-up times. They consider semidiscrete schemes ([14]) and totally discrete schemes using Euler and Runge-Kutta methods ([4]).

Other works that deserve being mentioned are $[5,15,21,27]$. The survey [6] summarizes the results contained in most of these articles.

The development and analysis of numerical methods for this kind of problems in several space dimensions are much less developed than the one dimensional case. In fact, numerical methods for this problem in dimension $d \geq 2$ with rigorous proofs of the their asymptotic properties are rare in the literature.

In this paper we introduce and analyze totally discrete explicit and semi-implicit methods for this problem in several space dimensions. For these methods we prove

- They reproduce the blow-up cases: if the continuous solution blows up in finite time, the same occurs with the numerical solution for small choices of the parameters of the method.

- They have the correct blow-up rate.

- The numerical blow-up times converge to the theoretical one (we can only prove an iterated limit and just for the explicit scheme).

- The localization of the numerical blow-up set.

As a first step to introduce the method we propose a method of lines: we discretize the space variable but the time variable $t$ remains continuous. In this stage we consider a general method with adequate assumptions. More precisely, we assume that for every $h>0$ small ( $h$ is the parameter of the method), there exists a set of nodes $\left\{x_{1}, \ldots, x_{N}\right\} \subset \bar{\Omega}(N=N(h))$, such that the numerical approximation of $u$ at the nodes $x_{k}$, is given by

$$
U(t)=\left(u_{1}(t), \ldots, u_{N}(t)\right) .
$$

That is, $u_{k}(t)$ stands for an approximation of $u\left(x_{k}, t\right)$. We assume that $U$ is the solution of the following ODE

$$
M U^{\prime}(t)=-A U(t)+M U^{p},
$$

with initial data given by $u_{k}(0)=u_{0}\left(x_{k}\right)$. In (1.2) and hereafter, all operations between vectors are understood coordinate by coordinate.

The precise assumptions on the matrices involved in the method are:

(P1) $M$ is a diagonal matrix with positive entries $m_{k}$.

(P2) $A$ is a nonnegative symmetric matrix, with nonpositive coefficients off the diagonal (i.e. $a_{k i} \leq 0$ if $k \neq i$ ) and $a_{k k}>0$.

(P3) $\sum_{i=1}^{N} a_{k i} \geq 0$. 
Taking into account (P1), the ODE (1.2) can be written as

$$
m_{k} u_{k}^{\prime}(t)=-\sum_{i=1}^{N} a_{k i} u_{i}(t)+m_{k} u_{k}^{p}(t), 1 \leq k \leq N,
$$

with initial data $u_{k}(0)=u_{0}\left(x_{k}\right)$.

As an example, we can consider a linear finite element approximation of problem (1.1) on a regular acute triangulation of $\Omega$ (see [12]). Let $V_{h}$ be the subspace of $H_{0}^{1}(\Omega)$ consisting of piecewise linear functions on the triangulation. The finite element approximation $u_{h}:\left[0, T_{h}\right) \rightarrow V_{h}$ verifies for each $t \in\left[0, T_{h}\right)$

$$
\int_{\Omega}\left(\left(u_{h}\right)_{t} v\right)^{I}=-\int_{\Omega} \nabla u_{h} \nabla v+\int_{\Omega}\left(\left(u_{h}\right)^{p} v\right)^{I},
$$

for every $v \in V_{h}$. Here $(\cdot)^{I}$ stands for the linear Lagrange interpolate at the nodes of the mesh. The vector $U(t)$, the values of $u_{h}(\cdot, t)$ at the nodes $x_{k}$, verifies a system like (1.2). In this case $M$ is the lumped mass matrix and $A$ is the stiffness matrix. The assumptions on the matrices $M$ and $A$ hold as we are considering an acute regular mesh. We observe that in this case $u_{h}=U^{I}$.

As another example, if $\Omega$ is a cube, $\Omega=(0,1)^{d}$, we can use a semidiscrete finite differences method to approximate the solution $u(x, t)$ obtaining an ODE system of the form (1.2).

We also need some kind of convergence result for the scheme, we will state the precise hypotheses concerning convergence in the statement of each theorem. Finally, in the Appendix we prove an $L^{\infty}$ convergence theorem under the consistency assumption. The possible convergence assumptions are

(H1) For every $\tau>0\left\|u-u_{h}\right\|_{L^{\infty}(\Omega \times[0, T-\tau])} \rightarrow 0$ as $h \rightarrow 0$.

(H2) $\left\|u-u_{h}\right\|_{H_{0}^{1}(\Omega)}(t) \rightarrow 0$ as $h \rightarrow 0$ for a.e. $t$

Once the ODE system is obtained, the next step is to discretize the time variable $t$. In [4] the authors suggest an adaptive in time step procedure to deal with the heat equation with a nonlinear boundary condition. They analyze explicit Euler and Runge-Kutta methods, however all these methods have to deal with restrictions in the time-step. In this work we first analyze an explicit Euler method and next we introduce a semi-implicit scheme in order to avoid the time-step restrictions. We use $U^{j}=\left(u_{1}^{j}, \ldots, u_{N}^{j}\right)$ for the values of the numerical approximation at time $t_{j}$, and $\tau_{j}=t_{j+1}-t_{j}$. When we consider the explicit scheme, $U^{j}$ is the solution of

$$
\begin{aligned}
& M U^{j+1}=M U^{j}+\tau_{j}\left(-A U^{j}+M\left(U^{j}\right)^{p}\right) \\
& U(0)=u_{0}^{I},
\end{aligned}
$$

or equivalently, if we denote $\partial u_{k}^{j+1}=\frac{1}{\tau_{j}}\left(u_{k}^{j+1}-u_{k}^{j}\right)$

$$
\begin{aligned}
& m_{k} \partial u_{k}^{j+1}=-\sum_{i=1}^{N} a_{k i} u_{k}^{j}+m_{k}\left(u_{k}^{j}\right)^{p}, 1 \leq i \leq N \\
& u_{k}^{0}=u_{0}\left(x_{k}\right), \quad 1 \leq k \leq N+1 .
\end{aligned}
$$


While for the implicit scheme $U^{j}$ is the solution of

$$
\begin{aligned}
& M U^{j+1}=M U^{j}-\tau_{j}\left(A U^{j+1}+M\left(U^{j}\right)^{p}\right) \\
& U(0)=u_{0}^{I} .
\end{aligned}
$$

Observe that (P1)-(P3) ensure that $\left(M+\tau_{j} A\right)^{-1}$ is well defined.

Also note that the scheme is not totally implicit since the nonlinear source $u^{p}$ is evaluated at time $t^{j}$ while the stiffness matrix $A$ is evaluated a time $t^{j+1}$. This mixture makes the scheme free of time-step restrictions while the explicit evaluation of $\left(U^{j}\right)^{p}$ avoids to solve a nonlinear system in each step that could lead to nonuniqunes or even to nonexistence (see [26]).

Now we choose the time steps $\tau_{j}=t_{j+1}-t_{j}$ in order to reproduce the asymptotic behavior. For different time-stepping strategies for this kind of problems see [1, 5, 11, 22, 23, 26]. We fix $\lambda$ small and take

$$
\tau_{j}=\frac{\lambda}{\left(w^{j}\right)^{p}}
$$

where

$$
w^{j}=\sum_{k=1}^{N} m_{k} u_{k}^{j}
$$

This choice for the time step is inspired by [4]. In that work the authors develop an adaptive procedure that adapts the time step in a similar way but using the maximum ( $L^{\infty}$-norm) instead of $w^{j}$ ( $L^{1}$-norm). In their problem the maximum is fixed at the right boundary node (i.e. $\left\|U^{j}\right\|_{\infty}=u_{N+1}^{j}$ ). In this problem, the maximum (the node $k$ such that $u_{k}^{j}=\left\|U^{j}\right\|_{\infty}$ ) can move from one node to another as $j$ increases. So the techniques used in [4] to study the behavior of $\left\|U^{j}\right\|_{\infty}$ do not apply here.

The motivation for this time-step is that, as will be shown, the behavior of $w^{j}$ is given by

$$
\partial w^{j+1} \sim\left(w^{j}\right)^{p} .
$$

Hence, with our selection of $\tau_{j}$ we can obtain

$$
w^{j+1} \sim w^{j}+\tau_{j}\left(w^{j}\right)^{p}=w^{j}+\lambda \sim w^{0}+(j+1) \lambda,
$$

and we obtain the asymptotic behavior of $w^{j}$, which is, as we will see, similar to the one for the continuous solution.

Then we study the asymptotic properties of the numerical schemes. We say that a solution of (1.3) (or (1.5)) blows up if

$$
\lim _{j \rightarrow \infty}\left\|U^{j}\right\|_{\infty}=\infty, \quad \text { and } \quad T_{h, \lambda}:=\sum_{j=1}^{\infty} \tau_{j}<\infty,
$$

we call $T_{h, \lambda}$ the numerical blow-up time. Here $\|\cdot\|_{\infty}$ stands for the usual infinity norm in $\mathbb{R}^{N}$.

To describe when the blow-up phenomena occurs in the discrete problem we introduce the following functional $\Phi_{h}: \mathbb{R}^{N} \rightarrow \mathbb{R}$. 


$$
\Phi_{h}(U) \equiv\langle A U, U\rangle-\left\langle\frac{1}{p+1} M U^{p+1}, M \mathbf{1}\right\rangle,
$$

where $\mathbf{1}=(1,1, \ldots, 1)^{\mathrm{T}}$. The functional $\Phi_{h}$ is a discrete version of

$$
\Phi(u)(t) \equiv \int_{\Omega} \frac{|\nabla u(s, t)|^{2}}{2} d s-\int_{\Omega} \frac{(u(s, t))^{p+1}}{p+1} d s .
$$

The functional $\Phi$ characterizes the solutions with blow-up in the continuous problem: in $[13,16]$ is proved that $u$ blows up at time $T$ if and only if $\Phi(u)(t) \rightarrow-\infty$ as $t \rightarrow T$. Here we prove a similar result for the discrete functional $\Phi_{h}$ and use this fact to prove that if the continuous solution has finite time blow-up, its numerical approximation also does when the parameters of the method are small enough.

Next we study the asymptotic behavior of the numerical approximations: if $U^{j}$ is a numerical solution with blow-up at time $T_{h, \lambda}$ its behavior is given by

$$
\left\|U^{j}\right\|_{\infty} \sim\left(T_{h, \lambda}-t^{j}\right)^{-1 /(p-1)} .
$$

Moreover, the numerical schemes reproduce the constant $C_{p}$ in the sense that

$$
\lim _{j \rightarrow \infty} \max _{1 \leq k \leq N} u_{k}^{j}\left(T_{h, \lambda}-t^{j}\right)^{1 /(p-1)}=C_{p}=\left(\frac{1}{p-1}\right)^{\frac{1}{p-1}} .
$$

The functional $\Phi_{h}$ is also useful to deal with the behavior of the numerical blowup times. Unfortunately we can only prove the convergence of an iterated limit,

$$
\lim _{h \rightarrow 0} \lim _{\lambda \rightarrow 0} T_{h, \lambda}=T .
$$

By means of the numerical blow-up rate we observe a propagation property for blow-up points. We prove that the nodes adjacent to those that blow-up as fast as the maximum may also blow-up (opposite to the continuous problem), but they did it with a slower rate. The number of adjacent nodes that also blow up is determined only by $p$ and is independent of $h$ and $\lambda$.

In other words, if we call $B^{*}(U)$ the set of nodes $k$ such that

$$
\lim _{j \rightarrow \infty} u_{k}^{j}\left(T_{h, \lambda}-t^{j}\right)^{1 /(p-1)}=C_{p},
$$

then the number of blow-up points that do not lie in $B^{*}(U)$ depends (explicitly) only on the power $p$.

We split the paper in two parts, the first one deals with the explicit scheme, in the second one the analysis for the implicit method is developed.

\section{THE EXPLICIT SCHEME}

The main tool in our proofs is a comparison argument, so we first prove a lemma which states that this comparison argument holds. Since we need restrictions in the time-step to prove this lemma they are essential for every result in this section. That is not the case for the implicit scheme. 
Definition 2.1. We say that $\left(Z^{j}\right)$ is a supersolution (resp.: subsolution) for (1.3) if verifies the equation with an inequality $\geq(\leq)$ instead of an equality.

Lemma 2.1. Assume the time step verifies

$$
\tau_{j}<\min _{1 \leq i \leq N} \frac{m_{i}}{a_{i i}} .
$$

Let $\left(\bar{U}^{j}\right),\left(\underline{U}^{j}\right)$ a super and a subsolution respectively for $(1.3)$ such that $\underline{U}^{0}<\bar{U}^{0}$, then $\underline{U}^{j}<\bar{U}^{j}$ for every $j$.

Proof: Let $Z^{j}=\bar{U}^{j}-\underline{U}^{j}$, by an approximation argument we can assume that we have strict inequalities in (1.3), then $\left(Z^{j}\right)$ verifies

$$
\begin{array}{ll}
M \partial Z^{j+1} & >-A Z^{j}+M\left(\left(\bar{U}^{j}\right)^{p}-\left(\underline{U}^{j}\right)^{p},\right. \\
Z^{0} & >0 .
\end{array}
$$

If the statement of the Lemma is false, then there exists a first time $t^{j+1}$ and a node $x_{k}$ such that $z_{k}^{j+1} \leq 0$. At that time we have

$$
z_{k}^{j+1}>\left(1-\tau_{j} \frac{a_{k k}}{m_{k}}\right) z_{k}^{j}+\tau_{j}\left(-\sum_{i \neq k} \frac{a_{k i}}{m_{k}} z_{i}^{j}+\left(\bar{u}_{k}^{j}\right)^{p}-\left(\underline{u}_{k}^{j}\right)^{p}\right) \geq 0,
$$

a contradiction.

2.1. Blow up in the numerical scheme. In this section we find conditions to guarantee blow-up in (1.4). We begin with some lemmas.

Since the matrix $A$ is symmetric (property (P2)), there exists a basis of eigenvectors for the following eigenvalue problem

$$
A \phi_{i}=\lambda_{i} M \phi_{i} .
$$

We call $\eta=\eta(h)$ the greatest eigenvalue of this problem, that is

$$
0 \leq \lambda_{i} \leq \eta(h), \quad 1 \leq i \leq N .
$$

Lemma 2.2. For every $y \in \mathbb{R}^{N}$ there holds

$$
\langle A y, y\rangle \leq \eta(h)\langle M y, y\rangle \text {. }
$$

Proof: As the matrix $M$ defines a scalar product in $\mathbb{R}^{N}$, we can assume that the eigenvectors $\phi_{i}$ are normalized such that

$$
\left\langle M \phi_{i}, \phi_{j}\right\rangle=\delta_{i j} .
$$

Let $y \in \mathbb{R}^{N}, y=\sum_{i=1}^{N} \alpha_{i} \phi_{i}$, then

$$
\begin{aligned}
\langle A y, y\rangle & =\left\langle\sum_{i=1}^{N} \alpha_{i} \lambda_{i} M \phi_{i}, \sum_{j=1}^{N} \alpha_{j} \phi_{j}\right\rangle \\
& =\sum_{i=1}^{N} \alpha_{i}^{2} \lambda_{i}\left\langle M \phi_{i}, \phi_{i}\right\rangle \\
& \leq \eta(h)\langle M y, y\rangle .
\end{aligned}
$$


Lemma 2.3. There exists a constant $\kappa$ that depends only on $h$ such that $U^{j_{0}} \geq \kappa$ for some $j_{0}$ implies that $\left(U^{j}\right)_{j \geq 1}$ blows up in finite time.

Remark 2.1. The constant $\kappa$ can be computed, in fact

$$
\kappa=\frac{\left(2 \sum_{i, k=1}^{N} a_{k i}\right)^{\frac{1}{p-1}}\left(\min _{k} m_{k}\right)^{\frac{p-2}{p-1}}}{\left(\sum_{k=1}^{N} m_{k}\right)^{p-1}} .
$$

Proof: Recall the definition of $w^{j}=\sum_{k=1}^{N} m_{k} u_{k}^{j}$, and observe that verifies

$$
\begin{aligned}
w^{j+1} & =w^{j}-\tau_{j} \sum_{k=1}^{N} \sum_{i=1}^{N} a_{k i} u_{i}^{j}+\tau_{j} \sum_{k=1}^{N} m_{k}\left(u_{k}^{j}\right)^{p} \\
& \geq w^{j}-\tau_{j} \sum_{i=1}^{N} u_{i}^{j} \sum_{k=1}^{N} a_{k i}+\tau_{j}\left(\sum_{k=1}^{N} m_{k}\right)^{1-p}\left(w^{j}\right)^{p} \\
& \geq w^{j}-\tau_{j}\left\|U^{j}\right\|_{\infty} \sum_{i, k=1}^{N} a_{k i}+\tau_{j}\left(\sum_{k=1}^{N} m_{k}\right)^{1-p}\left(w^{j}\right)^{p} \\
& =w^{j}+\tau_{j}\left(-c_{1} w^{j}+c_{2}\left(w^{j}\right)^{p}\right),
\end{aligned}
$$

where $c_{1}=\frac{\sum_{i, k=1}^{N} a_{k i}}{\min m_{k}}$.

So if $w^{j_{0}} \geq\left(\frac{2 c_{1}}{c_{2}}\right)^{1 /(p-1)}$ we have

$$
\begin{aligned}
w^{j_{0}+1} & \geq w^{j_{0}}+\frac{c_{2}}{2} \tau_{j}\left(w^{j_{0}}\right)^{p} \\
& =w^{j_{0}}+\frac{c_{2}}{2} \lambda .
\end{aligned}
$$

Applying this inequality inductively we obtain for $j \geq j_{0}$

$$
w^{j} \geq w^{j_{0}}+c \lambda\left(j-j_{0}\right) .
$$

Hereafter $c, c_{i}, C, C_{i}$, etc. are constants that may depend on $h$ but do not depend on $\lambda$ or the time variables. They may change from one line to another in the course of the proofs.

We have proved $w^{j} \rightarrow \infty$ as $j \rightarrow \infty$. It remains to check that $\sum \tau_{j}<\infty$, to do that we observe that

and

$$
\tau_{j}=\frac{\lambda}{\left(w^{j}\right)^{p}} \leq \frac{\lambda}{\left(w^{j_{0}}+c \lambda\left(j-j_{0}\right)\right)^{p}},
$$

$$
\sum_{j=j_{0}}^{\infty} \frac{\lambda}{\left(w^{j_{0}}+c \lambda\left(j-j_{0}\right)\right)^{p}} \leq \int_{0}^{\infty} \frac{\lambda}{\left(w^{j_{0}}+c \lambda s\right)^{p}} d s<\infty .
$$

This completes the proof.

Remark 2.2. In the course of the proof of the above Lemma we also proved $w^{j} \geq c j$ for $j$ large enough.

Now we are going to prove the reverse inequality to obtain the asymptotic behavior of $\left\|U^{j}\right\|_{\infty}$. 
Lemma 2.4. If $\left(U^{j}\right)$ is unbounded then

$$
\left\|U^{j}\right\|_{\infty} \sim w^{j} \sim j
$$

Proof: The relation $\left\|U^{j}\right\|_{\infty} \sim w^{j}$ holds since they define equivalent norms in $\mathbb{R}^{N}$. So we just have to prove $w^{j} \leq C j$. Observe that

$$
\begin{aligned}
w^{j+1} & =w^{j}-\tau_{j} \sum_{i=1}^{N} \sum_{k=1}^{N} a_{k i} u_{i}^{j}+\tau_{j} \sum_{i=1}^{N} m_{k}\left(u_{k}^{j}\right)^{p} \\
& \leq w^{j}+\tau_{j} \sum_{k=1}^{N} m_{k}\left(u_{k}^{j}\right)^{p} \\
& \leq w^{j}+C \tau_{j}\left(w^{j}\right)^{p} \\
& =w^{j}+C \lambda .
\end{aligned}
$$

Proceeding as before we get $w^{j} \leq w^{0}+C \lambda j \leq C j$, as we wanted to prove.

Theorem 2.1. Assume the time step $\tau_{j}$ verifies the restriction

$$
\tau_{j}<\frac{2}{p\left(w^{j+1}\right)^{p-1}+\eta(h)} .
$$

Then positive solutions of (1.4) blow up if there exists $j_{0}$ such that $\Phi_{h}\left(U^{j_{0}}\right)<0$.

Remark 2.3. We remark that the condition $\Phi_{h}\left(U^{j_{0}}\right)<0$ is similar to the one for the blow-up phenomena in the continuous problem, in fact for the continuous problem $u$ blows up if and only if $\Phi(u)\left(t_{0}\right)<0$ for some $t_{0} \geq 0$.

Proof: First we observe that $\Phi_{h}\left(U^{j}\right)$ decreases with $j$, in order to do that we take inner product of (1.3) with $U^{j+1}-U^{j}$ to obtain

$$
\begin{aligned}
0 & =\left\langle\frac{1}{\tau_{j}} M\left(U^{j+1}-U^{j}\right)+A U^{j}-M\left(U^{j}\right)^{p}, U^{j+1}-U^{j}\right\rangle \\
& =\tau_{j}\left\langle M \partial U^{j+1}, \partial U^{j+1}\right\rangle+\Phi_{h}\left(U^{j+1}\right)-\Phi_{h}\left(U^{j}\right)-\frac{1}{2}\left\langle A U^{j+1}, U^{j+1}\right\rangle \\
& +\left\langle A U^{j}, U^{j+1}\right\rangle-\frac{1}{2}\left\langle A U^{j}, U^{j}\right\rangle-\frac{1}{2}\left\langle M p\left(\xi^{j}\right)^{p-1},\left(U^{j+1}-U^{j}\right)^{2}\right\rangle .
\end{aligned}
$$

Hence we obtain,

$$
\begin{aligned}
& \Phi_{h}\left(U^{j+1}\right)-\Phi_{h}\left(U^{j}\right) \\
& \quad \leq \tau_{j}\left(\tau_{j} \frac{p\left(w^{j+1}\right)^{p-1}}{2}-1\right)\left\langle M \partial U^{j+1}, \partial U^{j+1}\right\rangle+\frac{\tau_{j}^{2}}{2}\left\langle A \partial U^{j+1}, \partial U^{j+1}\right\rangle \\
& \quad \leq \tau_{j}\left(\tau_{j} \frac{p\left(w^{j+1}\right)^{p-1}}{2}+\frac{\eta(h) \tau_{j}}{2}-1\right)\left\langle M \partial U^{j+1}, \partial U^{j+1}\right\rangle \leq 0,
\end{aligned}
$$

due to Lemma 2.2 and the restriction in the time step $\tau_{j}(2.1)$. Actually $\Phi_{h}\left(U^{j+1}\right)<$ $\Phi_{h}\left(U^{j}\right)$ unless $\left(U^{j}\right)$ is independent of $j$. So, $\Phi_{h}$ is a Lyapunov functional for (1.3). 
Next we observe that the steady states of (1.3) have positive energy. Let $\left(W^{j}\right)=W$ be a stationary solution of (1.3), then

$$
0=-A W+M W^{p} .
$$

Multiplying by $W / 2$ we obtain,

$$
\begin{aligned}
0 & =-\frac{1}{2}\langle A W, W\rangle+\frac{p+1}{2} \frac{1}{p+1}\left\langle M W^{p}, W\right\rangle \\
& \geq-\Phi_{h}(W) .
\end{aligned}
$$

Assume $\left(U^{j}\right)_{j \geq 1}$ is a bounded solution of (1.3), then there exists a subsequence that we still denote $\left(U^{j}\right)$ that converges to a steady state $W$ with positive energy.

As $\Phi_{h}\left(U^{j}\right)$ decreases and there exists $j_{0}$ such that $\Phi_{h}\left(U^{j_{0}}\right)<0$ then $\Phi_{h}(W)<0$, a contradiction. We conclude that $\left(U^{j}\right)$ is unbounded and (Lemma 2.3) has finite time blow-up.

Corollary 2.1. Assume the time-step restriction of the above theorem and the convergence hypotheses (H1), (H2). Let $u_{0}$ an initial data for (1.1) such that $u$ blows up in finite time $T$. Then $\left(U^{j}\right)$ blows up in finite time $T_{h, \lambda}$ for every $h$, $\lambda=\lambda(h)$ small enough. Moreover

$$
\lim _{h \rightarrow 0} \lim _{\lambda \rightarrow 0} T_{h, \lambda}=T
$$

Remark 2.4. If the fully-discrete method converges in $H_{0}^{1}(\Omega)$ a.e. $t$ then $\lambda$ can be chosen independent of $h$.

Proof: If $u$ blows up in finite time $T$ then (see $[13,16]$ )

$$
\Phi(u)(t) \equiv \int_{\Omega} \frac{|\nabla u(s, t)|^{2}}{2} d s-\int_{\Omega} \frac{(u(s, t))^{p+1}}{p+1} d s \rightarrow-\infty \quad(t \nearrow T) .
$$

Hence we have $\Phi(u)\left(t_{0}\right)<0$ for some $t_{0}<T$. Let $j_{0}=j_{0}(h, \lambda)$ be the first $j$ such that $t^{j_{0}} \geq t_{0}$. Note that the existence of $j_{0}$ is guaranteed by the convergence theorem for small $h, \lambda$ (see the Appendix). Now we use the convergence of $\left(U^{j}\right)$ to $u_{h}$ in $\left[0, t_{0}\right]$ and $(\mathrm{H} 1)$ to see that

$$
\lim _{h \rightarrow 0} \lim _{\lambda \rightarrow 0} \Phi_{h}\left(U^{j_{0}}\right)=\Phi(u)\left(t_{0}\right) .
$$

So for $h, \lambda=\lambda(h)$ small enough we get $\Phi_{h}\left(U^{j_{0}}\right)<0$ and so, by the above theorem $\left(U^{j}\right)$ blows up.

Now we turn our attention to the blow-up times. In [18] it is proved that the blow-up time of the semi-discrete solutions (solutions of (1.2)), that we denote $T_{h}$, converges to $T$ as $h \rightarrow 0$. That work deals just with $\Omega=(0,1)$ and a finite element method but the arguments are very similar. We sketch the proof for the sake of completeness.

Using similar arguments to the ones above, it can be seen that if the continuous solution blows up then for every $h$ small enough the semidiscrete solution $U(t)$ also does. Hence we can assume that the semidiscrete solution $U(t)$ is large enough in order to verify 


$$
\begin{gathered}
\frac{d}{d t}\langle M U(t), U(t)\rangle=2\left\langle M U^{\prime}(t), U(t)\right\rangle= \\
2\langle-A U(t), U(t)\rangle+2\left\langle M U^{p}(t), U(t)\right\rangle= \\
-4 \Phi_{h}(U(t))+\frac{2(p-1)}{p+1}\left\langle M U^{p}(t), U(t)\right\rangle \geq \\
4\left|\Phi_{h}(U(t))\right|+\frac{2(p-1)}{p+1}(\langle M U(t), U(t)\rangle)^{\frac{p+1}{2}} .
\end{gathered}
$$

Integrating between $t_{0}$ and $T_{h}$ we obtain

$$
\left(T_{h}-t_{0}\right) \leq \frac{C}{\left(-\Phi_{h}\left(U\left(t_{0}\right)\right)\right)^{\frac{p-1}{p+1}}} .
$$

Here $C$ depends only on $p$.

Given $\varepsilon>0$, we can choose $L$ large enough to ensure that

$$
\left(\frac{C}{L^{\frac{p-1}{p+1}}}\right) \leq \frac{\varepsilon}{2}
$$

As $u$ blows up at time $T$ we can choose $\tau<\frac{\varepsilon}{2}$ such that

$$
-\Phi(u(\cdot, T-\tau) \geq 2 L .
$$

If $h$ is small enough,

$$
-\Phi_{h}(U(T-\tau)) \geq L
$$

and hence

$$
T_{h}-(T-\tau) \leq\left(\frac{C}{\left(-\Phi_{h}(U(T-\tau))\right)^{\frac{p-1}{p+1}}}\right) \leq\left(\frac{C}{L^{\frac{p-1}{p+1}}}\right) \leq \frac{\varepsilon}{2} .
$$

Therefore,

$$
\left|T_{h}-T\right| \leq\left|T_{h}-(T-\tau)\right|+|\tau|<\varepsilon .
$$

We have proved $\lim _{h \rightarrow 0} T_{h}=T$, so we just have to prove that for fixed $h$

$$
\lim _{\lambda \rightarrow 0} T_{h, \lambda}=T_{h} .
$$

To do that we observe that from Lemma 2.3 there exists $j_{0}$, that does not depend on $\lambda$ such that for $j \geq j_{0}$

$$
w^{j} \geq w^{j_{0}}+c \lambda\left(j-j_{0}\right)
$$

hence

$$
\begin{aligned}
T_{h, \lambda}-t^{j} & =\sum_{l=j+1}^{\infty} \tau_{l}=\sum_{l=j+1}^{\infty} \frac{\lambda}{\left(w^{l}\right)^{p}} \\
& \leq \sum_{l=j+1}^{\infty} \frac{\lambda}{\left(w^{j_{0}}+c \lambda\left(l-j_{0}\right)\right)^{p}} \leq \int_{j}^{\infty} \frac{\lambda}{\left(w^{j_{0}}+c \lambda\left(s-j_{0}\right)\right)^{p}} d s \\
& =\frac{1}{c} \int_{w^{j_{0}}+c \lambda\left(j-j_{0}\right)}^{\infty} \frac{1}{s^{p}} d s \leq \frac{1}{c} \int_{w^{j_{0}}}^{\infty} \frac{1}{s^{p}} d s .
\end{aligned}
$$

This holds for any $j_{0}$ large enough and for every $j \geq j_{0}$. In particular we get 


$$
T_{h, \lambda}-t^{j} \leq \frac{1}{c} \int_{w^{j}}^{\infty} \frac{1}{s^{p}} d s
$$

This inequality is very useful since gives a bound (independent of $\lambda$ ) for the distance to the numerical blow-up time in terms of $w^{j}$. Hence, given $\varepsilon>0$ we can choose $K$ large enough in order to have

$$
\frac{1}{c} \int_{K}^{\infty} \frac{1}{s^{p}} d s \leq \frac{\varepsilon}{3}, \quad K^{-p}<\frac{\varepsilon}{3} .
$$

Next we choose $\tau<\frac{\varepsilon}{3}$ such that $\sum m_{k} u_{k}\left(T_{h}-2 \tau\right) \geq 2 K$ (recall that the vector $\left(u_{1}(t), \ldots, u_{N}(t)\right)$ is the solution of the semidiscrete scheme). For $\lambda=\lambda(h, \tau)$ small enough we get, from (H2), that $w^{j} \geq K$ for every $j$ such that $T_{h}-2 \tau \leq t^{j} \leq T_{h}-\tau$. We choose one of those $j$ and compute

$$
\begin{aligned}
\left|T_{h, \lambda}-T_{h}\right| & \leq\left|T_{h, \lambda}-t^{j}\right|+\left|t^{j}-T_{h}\right| \\
& \leq \frac{1}{c} \int_{K}^{\infty} \frac{1}{s^{p}} d s+2 \tau \\
& \leq \varepsilon
\end{aligned}
$$

This completes the proof.

2.2. Blow-up rate. In this section we study the asymptotic behavior of numerical solutions with blow-up.

Theorem 2.2. Let $u_{h, \lambda}$ a discrete solution with numerical blow-up at time $T_{h, \lambda}$, then

$$
\left\|U^{j}\right\|_{\infty} \sim\left(T_{h, \lambda}-t^{j}\right)^{-1 /(p-1)}
$$

Furthermore

$$
\lim _{j \rightarrow \infty}\left\|U^{j}\right\|_{\infty}\left(T_{h, \lambda}-t^{j}\right)^{1 /(p-1)}=C_{p}=\left(\frac{1}{p-1}\right)^{1 /(p-1)} .
$$

We want to remark that this is the behavior of the continuous solutions with blow-up.

Proof: We know from Lemma 2.3 that $w^{j}=\sum m_{k} u_{k}^{j}$ verifies

$$
w^{j+1} \geq w^{j}+c \lambda,
$$

so we have

$$
\begin{aligned}
\left(T_{h, \lambda}-t^{j}\right) & =\sum_{k=j+1}^{\infty} \tau_{j}=\sum_{k=j+1}^{\infty} \frac{\lambda}{\left(w^{j}\right)^{p}} \\
& \leq \int_{j}^{\infty} \frac{\lambda}{(w(s))^{p}} d s .
\end{aligned}
$$


Here $w(s)$ is the linear interpolant of $w^{j}\left(w(j)=w^{j}\right)$, hence for $j<s<j+1$ we have $w^{\prime}(s)=w^{j+1}-w^{j} \geq c \lambda$, and so

$$
\int_{j}^{\infty} \frac{\lambda}{(w(s))^{p}} d s \leq \int_{w^{j}}^{\infty} \frac{\lambda}{c v^{p} \lambda} d v \leq \frac{1}{c(p-1)}\left(\frac{1}{w^{j}}\right)^{p-1},
$$

or equivalently

$$
\left\|U^{j}\right\|_{\infty} \leq C w^{j} \leq C\left(T_{h, \lambda}-t^{j}\right)^{-1 /(p-1)}
$$

The reverse inequalities can be handled in a similar way to obtain

$$
\left\|U^{j}\right\|_{\infty} \sim w^{j} \sim\left(T_{h, \lambda}-t^{j}\right)^{-1 /(p-1)} .
$$

Next we look for the constant $C_{p}$ in the asymptotic behavior of the numerical solution, to do that we change variables. Let $\left(Y^{j}\right)$ be defined by

$$
y_{k}^{j}=u_{k}^{j}\left(T_{h, \lambda}-t^{j}\right)^{1 /(p-1)} \quad 1 \leq k \leq N .
$$

In the sequel of the proof we will use $\Delta y_{k}^{j+1}$ to denote

$$
\frac{y_{k}^{j+1}-y_{k}^{j}}{\tau_{j} /\left(T_{h, \lambda}-t^{j}\right)}
$$

This can be thought as $\tau_{j} /\left(T_{h, \lambda}-t^{j}\right)$ to be the time step in the new variables. With this notation the new variables verify

$$
\begin{aligned}
m_{k} \Delta y_{k}^{j+1}= & -\frac{\left(T_{h, \lambda}-t^{j+1}\right)^{\frac{1}{p-1}}}{\left(T_{h, \lambda}-t^{j}\right)^{\frac{1}{p-1}}}\left(T_{h, \lambda}-t^{j}\right) \sum_{i=1}^{N} a_{k i} y_{i}^{j} \\
& +m_{k} \frac{\left(T_{h, \lambda}-t^{j+1}\right)^{\frac{1}{p-1}}}{\left(T_{h, \lambda}-t^{j}\right)^{\frac{1}{p-1}}}\left(y_{k}^{j}\right)^{p} \\
& +\frac{\left(T_{h, \lambda}-t^{j}\right) m_{k} u_{k}^{j}}{\tau_{j}}\left(\left(T_{h, \lambda}-t^{j+1}\right)^{\frac{1}{p-1}}-\left(T_{h, \lambda}-t^{j}\right)^{\frac{1}{p-1}}\right), \\
y_{k}^{0}= & \left(T_{h, \lambda}\right)^{1 /(p-1)} u_{0}\left(x_{k}\right), \quad 1 \leq k \leq N+1 .
\end{aligned}
$$

We want to prove that $\left\|Y^{j}\right\|_{\infty} \rightarrow C_{p}$. To do that, we first observe that

$$
\lim _{j \rightarrow \infty} \frac{T_{h, \lambda}-t^{j}}{T_{h, \lambda}-t^{j+1}}=1
$$

since

$$
1 \leq \frac{T_{h, \lambda}-t^{j}}{T_{h, \lambda}-t^{j+1}}=\frac{\sum_{k=j+1}^{\infty} \tau_{k}}{\sum_{k=j+2}^{\infty} \tau_{k}}=1+\frac{\tau_{j+1}}{\sum_{k=j+2}^{\infty} \tau_{k}} \leq 1+\frac{\lambda /\left(w^{j+1}\right)^{p}}{C /\left(w^{j+1}\right)^{p-1}} \rightarrow 1 .
$$

Now assume there exists $\varepsilon>0$ and a subsequence that we still denote $\left(y_{k}^{j}\right)$ such that $y_{k}^{j}>C_{p}+\varepsilon$ for some $k=k(j)$. Then for those $y_{k}^{j}$ we have 


$$
\left(y_{k}^{j}\right)^{p}-\frac{1}{p-1} y_{k}^{j}>\frac{3 \delta}{m_{k}} .
$$

We also know from the blow-up rate that the new variables $y_{i}^{j}$ are bounded and so, we obtain for $j$ large enough

$$
\begin{aligned}
m_{k} \Delta y_{k}^{j+1} & \geq-\delta+m_{k}\left(\left(y_{k}^{j}\right)^{p}-\frac{1}{p-1} y_{k}^{j}\right)+m_{k}\left(y_{k}^{j}\right)^{p}\left(\frac{\left(T_{h, \lambda}-t^{j+1}\right)^{\frac{1}{p-1}}}{\left(T_{h, \lambda}-t^{j}\right)^{\frac{1}{p-1}}}-1\right) \\
& \geq \delta .
\end{aligned}
$$

This means that actually $y_{k}^{j}>C_{p}+\varepsilon$ for every $j$ large and consequently (2.2) is verified for all those $j$. So $y_{k}^{j}$ is unbounded, a contradiction.

If we assume $y_{k}^{j}<C_{p}-\varepsilon$ arguing along the same lines we obtain that $y_{k}^{j}$ verifies

$$
\begin{aligned}
m_{k} \Delta y_{k}^{j+1} \leq & \delta+m_{k} \frac{\left(T_{h, \lambda}-t^{j+1}\right)^{\frac{1}{p-1}}}{\left(T_{h, \lambda}-t^{j}\right)^{\frac{1}{p-1}}}\left(\left(y_{k}^{j}\right)^{p} \frac{1}{p-1} y_{k}^{j}\right) \\
& +\frac{m_{k}}{p-1} y_{k}^{j}\left(\frac{\left(T_{h, \lambda}-t^{j+1}\right)^{\frac{1}{p-1}}}{\left(T_{h, \lambda}-t^{j}\right)^{\frac{1}{p-1}}}-\frac{\left(T_{h, \lambda}-\xi^{j}\right)^{\frac{1}{p-1}-1}}{\left(T_{h, \lambda}-t^{j}\right)^{\frac{1}{p-1}-1}}\right) \\
\leq & 2 \delta+C\left(\left(y_{k}^{j}\right)^{p}-\frac{1}{p-1} y_{k}^{j}\right) .
\end{aligned}
$$

This shows that either $y_{k}^{j} \rightarrow 0$ as $j \rightarrow \infty$ or $m_{k} \Delta y_{k}^{j+1}<-\delta$, which means that $y_{k}^{j}$ is not bounded from below (this is not possible).

We conclude that if $y_{k}^{j}$ does not converge to zero, then converges to $C_{p}$. As the blow-up rate implies that for every $j$

$$
\left\|Y^{j}\right\|_{\infty} \geq c
$$

we have

$$
\lim _{j \rightarrow \infty}\left\|Y^{j}\right\|_{\infty}=\lim _{j \rightarrow \infty}\left\|U^{j}\right\|_{\infty}\left(T_{h, \lambda}-t^{j}\right)^{1 /(p-1)}=C_{p},
$$

as we wanted to prove.

2.3. Blow-up set. Now we turn our attention to the blow-up set. We consider the set $B^{*}(U)$ composed of those nodes that blow-up like $\left\|U^{j}\right\|_{\infty}$, that is

$$
B^{*}(U)=\left\{k: \lim _{j \rightarrow \infty} u_{k}^{j}\left(T_{h, \lambda}-t^{j}\right)^{1 /(p-1)}=C_{p}\right\} .
$$

We study the behavior of the nodes adjacent to this set, then we repeat the procedure with these last nodes.

Definition 2.2. We define $\mathcal{G}$ to be the graph with vertices in the nodes and we say that two different nodes are connected if and only if $a_{i j} \neq 0$. We consider the usual distance between nodes measured as a graph, see [19]. Finally, we denote by $d(k)$ the distance of the node $x_{k}$ to $B^{*}(U)$. 
We prove that $u_{k}^{j}$ blows up if and only if $d(k) \leq K$, where $K$ depends only on $p$.

Theorem 2.3. Assume the time step verifies $\tau_{j}<\frac{m_{i}}{a_{i i}},(1 \leq i \leq N)$. Then the blowup propagates outside $B^{*}(U)$ in the following way: let $K=\left[\frac{1}{p-1}\right]$, the solution of (1.3) blows up exactly at $K$ nodes near $B^{*}(U)$. More precisely,

$$
u_{k}^{j} \rightarrow+\infty \quad \Longleftrightarrow \quad d(k) \leq K .
$$

Moreover, if $d(k) \leq K$, the asymptotic behavior of $u_{k}^{j}$ is given by

$$
u_{k}^{j} \sim\left(T_{h, \lambda}-t^{j}\right)^{-\frac{1}{p-1}+d(k)},
$$

if $d(k) \neq \frac{1}{p-1}$ and if $d(k)=\frac{1}{p-1}$

$$
u_{k}^{j} \sim \ln \left(T_{h, \lambda}-t^{j}\right)
$$

Proof of Theorem 1.4 We want to show that the blow-up propagates $K$ nodes around $B^{*}(U)$, we begin with a node $x_{k}$ such that $d(k)=1$. We claim that the behavior of $u_{k}^{j}$ is given by

$$
u_{k}^{j} \sim\left\{\begin{array}{lll}
j^{-p+2} & \text { if } & p<2 \\
\ln j & \text { if } & p=2 \\
C & \text { if } & p>2
\end{array}\right.
$$

to prove that we will show that

$$
w_{A}^{j}=A \sum_{s=1}^{j} s \tau_{s-1},
$$

which has the behavior described above, can be used as super and subsolution for an equation verified by $u_{k}^{j}$ choosing $A$ appropriately.

We observe that $u_{k}^{j}$ satisfies

$$
\begin{aligned}
m_{k} \partial u_{k}^{j+1} & =-\sum_{i=1}^{N} a_{k i} u_{i}^{j}+m_{k}\left(u_{k}^{j}\right)^{p} \\
& \sim \Gamma_{1}\left\|U^{j}\right\|_{\infty}-\frac{a_{k k}}{m_{k}} u_{k}^{j}+\Gamma_{2}\left(u_{k}^{j}\right)^{p},
\end{aligned}
$$

for some constants $\Gamma_{i}$. In other words, there exists constants $c_{i}, C_{i}>0, i=1,2$ such that for $j$ large enough

$$
\partial u_{k}^{j+1} \leq C_{1} j-\frac{a_{k k}}{m_{k}} u_{k}^{j}+C_{2}\left(u_{k}^{j}\right)^{p}
$$

and

$$
\partial u_{k}^{j+1} \geq c_{1} j-\frac{a_{k k}}{m_{k}} u_{k}^{j}+c_{2}\left(u_{k}^{j}\right)^{p}
$$

Now we observe that if $A$ and $j$ are large enough, $w_{A}^{j}$ verifies

$$
\begin{aligned}
\partial w_{A}^{j} & =A(j+1) \\
& \geq C_{1} j-\frac{a_{k k}}{m_{k}} w_{A}^{j}+C_{2}\left(w_{A}^{j}\right)^{p},
\end{aligned}
$$


since $\left(w_{A}^{j}\right)^{p} / j \rightarrow 0$ as $j$ goes to infintiy. Hence $w_{A}^{j}$ is a supersolution for (2.3) and so

$$
u_{k}^{j} \leq w_{A}^{j}
$$

(a comparison principle like Lemma 2.2 holds for this equation and can be proved in the same way) On the other hand if we choose $A$ small we get

$$
\begin{aligned}
\partial w_{A}^{j} & =A(j+1) \\
& \leq c_{1} j-\frac{a_{k k}}{m_{k}} w_{A}^{j}+c_{2}\left(w_{A}^{j}\right)^{p},
\end{aligned}
$$

Hence now we can use $w_{A}^{j}$ as a subsolution for (2.4) to handle the other inequality. Therefore

$$
u_{k}^{j} \sim w_{A}^{j} .
$$

We observe that if $p<2$ the node $x_{k}$ is a blow-up node and we also have the blow-up rate for this node $\left(u_{k}^{j} \sim j^{-p+2}\right)$. If $p>2$ this node is bounded. Next we assume $p<2$ (if $p>2$ it is easy to prove that every node $k$ with $d(k) \geq 1$ is bounded) and we are going to find the behavior of a node, that we still denote $k$, such that $d(k)=2$. That is, it is not adyacent to $B^{*}(U)$ and it is adyacent to a node wich has the behavior $j^{-p+2}$.

Now let

$$
w_{A}^{j}=A \sum_{s=1}^{j} \tau_{s} s^{-p+2}
$$

and observe that $u_{k}^{j}$ verifies

$$
m_{k} \partial u_{k}^{j+1}=-\sum_{i=1}^{N} a_{k i} u_{i}^{j}+m_{k}\left(u_{k}^{j}\right)^{p} \sim \Gamma_{1} j^{-p+2}-\frac{a_{k k}}{m_{k}} u_{k}^{j}+\Gamma_{2}\left(u_{k}^{j}\right)^{p} .
$$

That is, for $j$ large we have

$$
\partial u_{k}^{j+1} \leq C_{1} j^{-p+2}-\frac{a_{k k}}{m_{k}} u_{k}^{j}+C_{2}\left(u_{k}^{j}\right)^{p}
$$

and

$$
\partial u_{k}^{j+1} \geq c_{1} j^{-p+2}-\frac{a_{k k}}{m_{k}} u_{k}^{j}+c_{2}\left(u_{k}^{j}\right)^{p}
$$

Now for $A$ and $j$ large, $w_{A}^{j}$ verifies

$$
\begin{aligned}
\partial w_{A}^{j} & =A(j+1)^{-p+2} \\
& \geq C_{1} j^{-p+2}-C_{2} w_{A}^{j}+C_{3}\left(w_{A}^{j}\right)^{p} .
\end{aligned}
$$

Hence $w_{A}^{j}$ is a supersolution for (2.5) and so

$$
u_{k}^{j} \leq w_{A}^{j} .
$$

On the other hand if we choose $A$ small we get

$$
\begin{aligned}
\partial w_{A}^{j} & =A(j+1)^{-p+2} \\
& \leq c_{1} j^{-p+2}-c_{2} w_{A}^{j}+c_{3}\left(w_{A}^{j}\right)^{p},
\end{aligned}
$$

Now we can use $w_{A}^{j}$ as a subsolution for (2.4) to handle the other inequality. So

$$
u_{k}^{j} \sim w_{A}^{j} \text {. }
$$


If $p<3 / 2$ the node $x_{k}$ is a blow-up node and we also have the blow-up rate for this node $\left(u_{k}^{j} \sim j^{-2 p+3}\right)$. If $p>3 / 2$ this node is bounded. In the case $p<3 / 2$ we repeat this procedure inductively to obtain the theorem.

\section{THE IMPLICIT SCHEME}

In order to avoid the time step restrictions we now consider the semi-implicit scheme (1.5) and prove that similar properties can be observed in this procedure.

Lemma 3.1. Let $\left(\bar{U}^{j}\right),\left(\underline{U}^{j}\right)$ a super and a subsolution respectively for (1.5) such that $\underline{U}^{0}<\bar{U}^{0}$, then $\underline{U}^{j}<\bar{U}^{j}$ for every $j$.

Proof: Let $Z^{j}=\bar{U}^{j}-\underline{U}^{j}$, we assume that we have strict inequalities in (1.5), then $\left(Z^{j}\right)$ verifies

$$
\begin{aligned}
M \partial Z^{j+1} & >-A Z^{j+1}+M\left(\left(\bar{U}^{j}\right)^{p}-\left(\underline{U}^{j}\right)^{p}\right), \\
Z^{0} & >0 .
\end{aligned}
$$

If the statement of the Lemma is false, then there exists a first time $t^{j+1}$ and a node $x_{k}$ with $z_{k}^{j+1}=\min _{1 \leq i \leq N} z_{i}^{j+1} \leq 0$ that verifies

$$
z_{k}^{j+1}>z_{k}^{j}-\tau_{j} \sum_{i=1}^{N} \frac{a_{k i}}{m_{k}} z_{i}^{j+1}+\tau_{j}\left(\left(\bar{u}_{k}^{j}\right)^{p}-\left(\underline{u}_{k}^{j}\right)^{p}\right) \geq z_{k}^{j}-\frac{\tau_{j} z_{k}^{j+1}}{m_{k}} \sum_{i=1}^{N} a_{k i} \geq 0,
$$

a contradiction.

\subsection{Blow-up in the numerical scheme.}

Lemma 3.2. There exists a constant $\kappa$ such that $U^{j_{0}} \geq \kappa$ implies that $U^{j}$ blows up in finite time. Furthermore

$$
\left\|U^{j}\right\|_{\infty} \sim w^{j} \sim j
$$

Remark 3.1. Unfortunately in this case we are not able to prove that the constant $\kappa$ does not depend on $\lambda$ and hence we can not prove the convergence of the numerical blow-up times.

Proof:

$$
\begin{aligned}
w^{j+1} & =w^{j}-\tau_{j} \sum_{k=1}^{N} \sum_{i=1}^{N} a_{k i} u_{i}^{j+1}+\tau_{j} \sum_{k=1}^{N} m_{k}\left(u_{k}^{j}\right)^{p} \\
& \leq w^{j}+\tau_{j} \sum_{k=1}^{N} m_{k}\left(u_{k}^{j}\right)^{p} \\
& \leq w^{j}+C \tau_{j}\left(w^{j}\right)^{p} \\
& =w^{j}+C \lambda .
\end{aligned}
$$

Hence $w^{j} \leq C j$. To prove the reverse inequality we observe that 


$$
\begin{aligned}
w^{j+1} & =w^{j}-\tau_{j} \sum_{k=1}^{N} \sum_{i=1}^{N} a_{k i} u_{i}^{j+1}+\tau_{j} \sum_{k=1}^{N} m_{k}\left(u_{k}^{j}\right)^{p} \\
& \geq w^{j}-\tau_{j} C_{1} w^{j+1}+\tau_{j} C_{2}\left(w^{j}\right)^{p}
\end{aligned}
$$

where

$$
C_{1}=\max _{1 \leq i \leq N} \frac{\sum_{k} a_{k i}}{m_{i}}, \quad C_{2}=\left(\sum_{k=1}^{N} m_{k}\right)^{1-p} .
$$

Hence

$$
\left(1+C_{1} \tau_{j}\right) w^{j+1} \geq w^{j}+C_{2} \tau_{j}\left(w^{j}\right)^{p} .
$$

Now we look for a subsolution of (3.2) of the form $z^{j}=\Gamma j$. This sequence verifies

$$
\left(1+C_{1} \tau_{j}\right) z^{j+1}=z^{j}+\Gamma C_{1} \tau_{j} j+\Gamma\left(1+C_{1} \tau_{j}\right) \leq z^{j}+C_{2} \tau_{j}\left(z^{j}\right)^{p}
$$

if $\Gamma \geq\left(\frac{\lambda C_{1}+C_{1}+1}{\lambda C 2}\right)^{1 /(p-1)}$ is small. As the discrete maximum principle holds for this equation we obtain

$$
w^{j} \geq z^{j}=\Gamma j .
$$

This completes the proof.

Theorem 3.1. If $\Phi_{h}\left(U^{j_{0}}\right)<0$ for some $j_{0}$, then $\left(U^{j}\right)_{j \geq 1}$ blows up.

Proof: We first observe that also for this scheme $\Phi_{h}\left(U^{j}\right)$ decreases with $j$. We take inner product of (1.5) with $U^{j+1}-U^{j}$

$$
\begin{aligned}
0= & \left\langle\frac{1}{\tau_{j}} M\left(U^{j+1}-U^{j}\right)+A U^{j+1}-M\left(U^{j}\right)^{p}, U^{j+1}-U^{j}\right\rangle \\
= & \tau_{j}\left\langle M \partial U^{j+1}, \partial U^{j+1}\right\rangle+\Phi_{h}\left(U^{j+1}\right)-\Phi_{h}\left(U^{j}\right)+\frac{1}{2}\left\langle A U^{j+1}, U^{j+1}\right\rangle \\
& -\left\langle A U^{j}, U^{j+1}\right\rangle+\frac{1}{2}\left\langle A U^{j}, U^{j}\right\rangle+\frac{p}{2}\left\langle M\left(\xi^{j}\right)^{p-1},\left(U^{j+1}-U^{j}\right)^{2}\right\rangle .
\end{aligned}
$$

Hence we obtain,

$$
\begin{aligned}
\Phi_{h}\left(U^{j+1}\right)-\Phi_{h}\left(U^{j}\right)= & -\tau_{j}\left\langle M \partial U^{j+1}, \partial U^{j+1}\right\rangle-\frac{\tau_{j}^{2}}{2}\left\langle A \partial U^{j+1}, \partial U^{j+1}\right\rangle \\
& -\frac{p}{2}\left\langle M\left(\xi^{j}\right)^{p-1},\left(U^{j+1}-U^{j}\right)^{2}\right\rangle \\
\leq & 0 .
\end{aligned}
$$

The steady states of (1.5) are the same of the ones for (1.3), so they have positive energy. Now, assume $\left(U^{j}\right)$ is a bounded solution of (1.5), then it has a convergent subsequece. Its limit $W$ is a steady state with positive energy.

As $\Phi_{h}\left(U^{j}\right)$ decreases and there exists $j_{0}$ with $\Phi_{h}\left(U^{j_{0}}\right)<0$ then $\Phi_{h}(W)<0$, a contradiction. We conclude that $\left(U^{j}\right)$ is unbounded and by Lemma 3.2 has finite time blow-up. 
Corollary 3.1. Assume the convergence hypotheses (H1), (H2). Let $u_{0}$ an initial data for (1.1) such that u blows up in finite time T. Then $u_{h, \lambda}$ blows up in finite time $T_{h, \lambda}$ for every $h, \lambda=\lambda(h)$ small enough.

Proof: If $u$ blows up in finite time $T$ then

$$
\Phi(u)(t) \equiv \int_{\Omega} \frac{|\nabla u(s, t)|^{2}}{2} d s-\int_{\Omega} \frac{(u(s, t))^{p+1}}{p+1} d s \rightarrow-\infty \quad(t \nearrow T) .
$$

Hence there exists a time $t_{0}<T$ with $\Phi(u)\left(t_{0}\right)<0$. Let $j_{0}=\inf \left\{j: t^{j} \geq t_{0}\right\}$. We use the convergence hypothesis $(\mathrm{H} 1)$ and the convergence of $\left(U^{j}\right)$ to $u_{h}$ in $\left[0, t_{0}\right]$ to see that

$$
\lim _{h \rightarrow 0} \lim _{\lambda \rightarrow 0} \Phi_{h}\left(U^{j_{0}}\right)=\Phi(u)\left(t_{0}\right) .
$$

So for $h, \lambda(h)$ small enough we get $\Phi_{h}\left(U^{j_{0}}\right)<0$ and so $\left(U^{j}\right)$ blows up.

Next we turn our attention to the blow-up rate of the discrete solutions.

\subsection{Blow-up rate.}

Theorem 3.2. Let $u_{h, \lambda}$ be a solution with blow-up at time $T_{h, \lambda}$, then

$$
\max _{1 \leq k \leq N} u_{k}^{j} \sim\left(T_{h, \lambda}-t^{j}\right)^{-1 /(p-1)} .
$$

Moreover

$$
\lim _{j \rightarrow \infty} \max _{1 \leq k \leq N} u_{k}^{j}\left(T_{h, \lambda}-t^{j}\right)^{1 /(p-1)}=C_{p}=\left(\frac{1}{p-1}\right)^{1 /(p-1)} .
$$

Proof: The first part of the proof is the same as the one for the explicit scheme so we assume we have proved

$$
\left\|U^{j}\right\|_{\infty} \sim\left(T_{h, \lambda}-t^{j}\right)^{-\frac{1}{p-1}},
$$

and we are going to prove the convergence of the selfsimilar variables $\left(Y^{j}\right)$ to $C_{p}$. Let $\left(Y^{j}\right), \Delta y_{k}^{j+1}$ be defined as in the previous section. In the semi-implicit scheme, these variables verify

$$
\begin{aligned}
m_{k} \Delta y_{k}^{j+1} & =-\left(T_{h, \lambda}-t^{j}\right) \sum_{i=1}^{N} a_{k i} y_{i}^{j+1}+m_{k} \frac{\left(T_{h, \lambda}-t^{j+1}\right)^{1 /(p-1)}}{\left(T_{h, \lambda}-t^{j}\right)^{1 /(p-1)}}\left(y_{k}^{j}\right)^{p} \\
& -m_{k} u_{k}^{j}\left(\left(T_{h, \lambda}-t^{j}\right)^{1 /(p-1)}-\left(T_{h, \lambda}-t^{j+1}\right)^{1 /(p-1)}\right), \\
y_{k}^{0} & =T_{h, \lambda}^{1 /(p-1)} u_{0}\left(x_{k}\right) .
\end{aligned}
$$

If we assume the existence of $\varepsilon>0$ and a subsequence such that $y_{k}^{j}>C_{p}+\varepsilon$ for some $k=k(j)$. Then for those $y_{k}^{j}$, as they are bounded, we have for $j$ large 


$$
\begin{aligned}
\Delta y_{k}^{j+1} \geq & -\delta+m_{k} \frac{\left(T_{h, \lambda}-t^{j+1}\right)^{1 /(p-1)}}{\left(T_{h, \lambda}-t^{j}\right)^{1 /(p-1)}}\left(\left(y_{k}^{j}\right)^{p}-\frac{1}{p-1} y_{k}^{j}\right) \\
& +\frac{1}{p-1} y_{k}^{j}\left[1-\frac{\left(T_{h, \lambda}-t^{j+1}\right)^{\frac{1}{p-1}}-1}{\left(T_{h, \lambda}-t^{j}\right)^{\frac{1}{p-1}-1}}\right] \\
\geq & \delta .
\end{aligned}
$$

Hence $y_{k}^{j}>C_{p}+\varepsilon$ for every $j$ large enough and consequently (3.3) is verified for all those $j$. So $y_{k}^{j}$ is unbounded, a contradiction.

The case where there exists an infinite number of $(j, k)$ with $y_{k}^{j}<C_{p}-\varepsilon$ can be handled in the same way to conclude that as $j \rightarrow \infty$ either $y_{k}^{j} \rightarrow 0$ or $y_{k}^{j} \rightarrow C_{p}$. Now we use the blow-up rate to obtain

$$
\lim _{j \rightarrow \infty} \max _{1 \leq k \leq N} y_{k}^{j}=\lim _{j \rightarrow \infty} \max _{1 \leq k \leq N}\left(T_{h, \lambda}-t^{j}\right)^{1 /(p-1)} u_{k}^{j}=C_{p},
$$

as we wanted to prove.

3.3. Blow-up set. The propagation property for the blow-up nodes holds for the implicit scheme and its proof is very similar. We do not include it.

\section{Appendix}

In this appendix we prove that if the general method considered for the space discretization is consistent (see below) then the totally discrete method converges in the $L^{\infty}$ norm. We perform the proofs for the explicit scheme, they can be extended to the implicit one.

Definition 4.1. Let $w$ be a regular solution of

$$
\begin{array}{ll}
w_{t}=\Delta w+f(x, t) & \text { in } \Omega \times(0, T), \\
w=0 & \text { on } \partial \Omega \times(0, T) .
\end{array}
$$

We say that the scheme (1.2) is consistent if for any $t \in(0, T-\tau)$ it holds

$$
m_{k} w_{t}\left(x_{k}, t\right)=-\sum_{i=1}^{N} a_{k i} w\left(x_{k}, t\right)+m_{k} f\left(x_{k}, t\right)+\rho_{k, h}(t),
$$

and there exists a function $\rho: \mathbb{R}_{+} \rightarrow \mathbb{R}_{+}$such that

$$
\max _{k} \frac{\left|\rho_{k, h}(t)\right|}{m_{k}} \leq \rho(h), \quad \text { for every } t \in(0, T-\tau),
$$

with $\rho(h) \rightarrow 0$ if $h \rightarrow 0$. The function $\rho$ is called the modulus of consistency of the method.

If we consider for example a finite differences scheme in a cube $\Omega=(0,1)^{d} \subset \mathbb{R}^{d}$. Then the modulus of consistency can be taken as $\rho(h)=C h^{2}$.

Theorem 4.1. Let $u$ be a regular solution of $(1.1)\left(u \in C^{2,1}(\bar{\Omega} \times[0, T-\tau])\right.$ and $\left(U^{j}\right)_{j \geq 1}$ the numerical approximation given by (1.4). If the scheme (1.2) is consistent with modulus of consistency $\rho$, then there exists positive constants $C, h_{0}, \lambda_{0}$ depending on $\|u\|$ in $C^{2,1}(\bar{\Omega} \times[0, T-\tau])$ such that for every $h<h_{0}, \lambda<\lambda_{0}$ holds 


$$
\max _{j} \max _{1 \leq k \leq N}\left|u_{k}^{j}-u\left(x_{k}, t_{j}\right)\right| \leq C(\rho(h)+\lambda) .
$$

\section{Proof:}

We define the error functions

$$
e_{k}^{j}=u\left(x_{k}, t_{j}\right)-u_{k}^{j} .
$$

By (4.1) and (1.4), these functions verify

$$
m_{k} \partial e_{k}^{j+1} \leq-\sum_{i=1}^{N} a_{k i} e_{i}^{j}+m_{k}\left(u^{p}\left(x_{k}, t_{j}\right)-\left(u_{k}^{j}\right)^{p}\right)+\rho_{k}(h)+C m_{k} \lambda,
$$

where $C$ is a bound for $\left\|u_{t t}\right\|_{L^{\infty}(\Omega \times[0, T-\tau])}$. Let

$$
t_{0}=\max \left\{t: t<T-\tau, \max _{i} \max _{t_{j}<t}\left|e_{i}^{j}\right| \leq 1\right\} .
$$

We will see by the end of the proof that $t_{0}=T-\tau$ for $h, \lambda$ small enough. In $\left[0, t_{0}\right]$ we have

$$
m_{k} \partial e_{k}^{j+1}=-\sum_{i=1}^{N} a_{k i} e_{i}^{j}+m_{k} p\left(\xi_{k}^{j}\right)^{p-1} e_{k}^{j}+\rho_{k}(h)+C m_{k} \lambda,
$$

hence, in $\left[0, t_{0}\right], E^{j}=\left(e_{1}^{j}, \ldots, e_{N}^{j}\right)$ satisfies

$$
\begin{array}{ll}
M \partial E^{j+1} & \leq-A E^{j}+K M E^{j}+(\rho(h)+C \lambda) M \mathbf{1}^{t} \\
E(0) & =0 .
\end{array}
$$

Here $K$ is the Lipchitz constant for $f(u)=u^{p}$ in $\left[0,\|u(\cdot, T-\tau)\|_{L^{\infty}}\right]$. Let us now define $W^{j}=\left(w_{1}^{j}, \ldots, w_{N}(t)\right)$, which will be used as a supersolution.

$$
w_{i}^{j}=e^{(2 K+1) t_{j}}(\rho(h)+C \lambda) .
$$

It is easy to check that $W^{j}$ verifies

$$
M \partial W^{j+1}>-A W^{j}+K M W^{j}+(\rho(h)+C \lambda) M 1^{t},
$$

Hence $W^{j}$ is a supersolution for (4.2), and by Lemma 2.1 we get

$$
e_{k}^{j} \leq e^{(2 K+1) t_{j}}(\rho(h)+C \lambda), \quad t_{j} \in\left[0, t_{0}\right] .
$$

Arguing along the same lines with $-E^{j}$, we obtain

$$
\left|e_{k}^{j}\right| \leq e^{(2 K+1) T}(\rho(h)+C \lambda) \leq C(\rho(h)+\lambda), \quad t_{j} \in\left[0, t_{0}\right] .
$$

Using this fact, since $\rho(h)$ goes to zero, we get that $\left|e_{k}^{j}\right| \leq 1$ for every $t_{j} \in[0, T-\tau]$ for every $h, \lambda$ small enough. Therefore $t_{0}=T-\tau$ for $h, \lambda$ small enough. This proves the convergence of the scheme. In fact we have that for every $h<h_{0}, \lambda<\lambda_{0}$

$$
\max _{j} \max _{1 \leq k \leq N}\left|u_{k}^{j}-u\left(x_{k}, t_{j}\right)\right| \leq C(\rho(h)+\lambda) .
$$

\section{Acknowledgments}

I would like to thank Julio D. Rossi for his help, his encouragement, and his friendship. 


\section{REFERENCES}

[1] L. M. Abia, J. C. López-Marcos, and J. Martínez. The Euler method in the numerical integration of reaction-diffusion problems with blow-up. Appl. Numer. Math., 38(3):287-313, 2001.

[2] Luis M. Abia, J. C. López-Marcos, and Julia Martínez. Blow-up for semidiscretizations of reaction-diffusion equations. Appl. Numer. Math., 20(1-2):145-156, 1996. Workshop on the method of lines for time-dependent problems (Lexington, KY, 1995).

[3] Luis M. Abia, J. C. López-Marcos, and Julia Martínez. On the blow-up time convergence of semidiscretizations of reaction-diffusion equations. Appl. Numer. Math., 26(4):399-414, 1998.

[4] G. Acosta, R. G. Durán, and J. D. Rossi. An adaptive time step procedure for a parabolic problem with blow-up. Computing, 68(4):343-373, 2002.

[5] C. Bandle and H. Brunner. Numerical analysis of semilinear parabolic problems with blow-up solutions. Rev. Real Acad. Cienc. Exact. Fís. Natur. Madrid, 88(2-3):203-222, 1994.

[6] Catherine Bandle and Hermann Brunner. Blowup in diffusion equations: a survey. J. Comput. Appl. Math., 97(1-2):3-22, 1998.

[7] Marsha Berger and Robert V. Kohn. A rescaling algorithm for the numerical calculation of blowing-up solutions. Comm. Pure Appl. Math., 41(6):841-863, 1988.

[8] C. J. Budd and G. J. Collins. An invariant moving mesh scheme for the nonlinear diffusion equation. In Proceedings of the International Centre for Mathematical Sciences Conference on Grid Adaptation in Computational PDEs: Theory and Applications (Edinburgh, 1996), volume 26, pages 23-39, 1998.

[9] Chris J. Budd, Shaohua Chen, and Robert D. Russell. New self-similar solutions of the nonlinear Schrödinger equation with moving mesh computations. J. Comput. Phys., 152(2):756-789, 1999.

[10] Chris J. Budd, Weizhang Huang, and Robert D. Russell. Moving mesh methods for problems with blow-up. SIAM J. Sci. Comput., 17(2):305-327, 1996.

[11] Yun Gang Chen. Asymptotic behaviours of blowing-up solutions for finite difference analogue of $u_{t}=u_{x x}+u^{1+\alpha}$. J. Fac. Sci. Univ. Tokyo Sect. IA Math., 33(3):541-574, 1986.

[12] Philippe G. Ciarlet. The finite element method for elliptic problems. North-Holland Publishing Co., Amsterdam, 1978. Studies in Mathematics and its Applications, Vol. 4.

[13] Carmen Cortázar, Manuel del Pino, and Manuel Elgueta. The problem of uniqueness of the limit in a semilinear heat equation. Comm. Partial Differential Equations, 24(11-12):21472172, 1999.

[14] R. G. Duran, J. I. Etcheverry, and J. D. Rossi. Numerical approximation of a parabolic problem with a nonlinear boundary condition. Discrete Contin. Dynam. Systems, 4(3):497506, 1998.

[15] Julián Fernández Bonder and Julio D. Rossi. Blow-up vs. spurious steady solutions. Proc. Amer. Math. Soc., 129(1):139-144, 2001.

[16] Yoshikazu Giga and Robert V. Kohn. Characterizing blowup using similarity variables. Indiana Univ. Math. J., 36(1):1-40, 1987.

[17] Yoshikazu Giga and Robert V. Kohn. Nondegeneracy of blowup for semilinear heat equations. Comm. Pure Appl. Math., 42(6):845-884, 1989.

[18] Pablo Groisman and Julio D. Rossi. Asymptotic behaviour for a numerical approximation of a parabolic problem with blowing up solutions. J. Comput. Appl. Math., 135(1):135-155, 2001.

[19] Frank Harary. Graph theory. Addison-Wesley Publishing Co., Reading, Mass.-Menlo Park, Calif.-London, 1969.

[20] Weizhang Huang, Yuhe Ren, and Robert D. Russell. Moving mesh partial differential equations (MMPDES) based on the equidistribution principle. SIAM J. Numer. Anal., 31(3):709730, 1994.

[21] Marie-Noëlle Le Roux. Semidiscretization in time of nonlinear parabolic equations with blowup of the solution. SIAM J. Numer. Anal., 31(1):170-195, 1994.

[22] Tomoyasu Nakagawa. Blowing up of a finite difference solution to $u_{t}=u_{x x}+u_{2} .$. Appl. Math. Optim., 2(4):337-350, 1975/76.

[23] Tomoyasu Nakagawa and Teruo Ushijima. Finite element analysis of the semi-linear heat equation of blow-up type. In John J. H. Miller, editor, Topics in numerical analysis. III, 
pages 275-291. Published for the Royal Irish Academy by Academic Press, London-New York, 1977.

[24] C. V. Pao. Nonlinear parabolic and elliptic equations. Plenum Press, New York, 1992.

[25] Alexander A. Samarskii, Victor A. Galaktionov, Sergei P. Kurdyumov, and Alexander P. Mikhailov. Blow-up in quasilinear parabolic equations, volume 19 of de Gruyter Expositions in Mathematics. Walter de Gruyter \& Co., Berlin, 1995. Translated from the 1987 Russian original by Michael Grinfeld and revised by the authors.

[26] A. M. Stuart and M. S. Floater. On the computation of blow-up. European J. Appl. Math., 1(1):47-71, 1990

[27] Takeo K. Ushijima. On the approximation of blow-up time for solutions of nonlinear parabolic equations. Publ. Res. Inst. Math. Sci., 36(5):613-640, 2000.

[28] J. J. L. Velázquez. Classification of singularities for blowing up solutions in higher dimensions. Trans. Amer. Math. Soc., 338(1):441-464, 1993.

Instituto de Cálculo, FCEyn, Universidad de Buenos Aires

Pabellón II Ciudad Universitaria (1428) Buenos Aires, Argentina.

E-mail address: pgroisma@dm.uba.ar 